
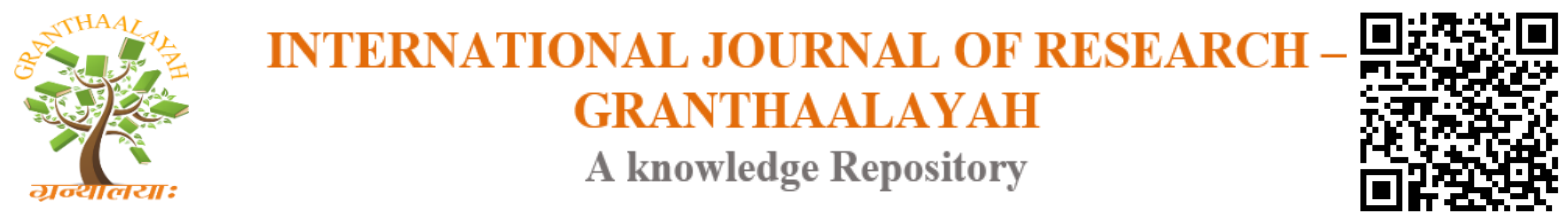

Science

\title{
WHAT IS THE MOST APPROPRIATE METHOD FOR COMMON FEMORAL ARTERY CANNULATION IN PATIENTS AGED ABOVE 75 YEARS WHICH IS THE MOST APPROPRIATE METHOD
}

\author{
Hasan Ata Bolayir *1, Hakan Gunes ${ }^{2}$, Muratkerkutluoglu ${ }^{2}$ \\ ${ }^{*}$ Sivas Numune State Hospital, Department of Cardiology, Sivas, Turkey \\ ${ }^{2}$ Kahramanmarassutcu Imam University, Medicine Faculty,Department of Cardiology, \\ Kahramanmaras, Turkey
}

\begin{abstract}
Background: Angiography is the gold standard in the diagnosis and treatment of coronary artery diseases, and the femoral approach is usually preferred. Here we aimed to determine which intervention technique minimizes complications of the femoral approach in patients aged $>75$ years.

Method: 309 patients aged $>75$ years were included. They were divided into group 1 (ultrasoundguided intervention group) and group 2 (palpation-guided intervention group) and were compared in terms of clinical and demographic characteristics, laboratory findings, surgical complications, average intervention time, success rate at initial intervention, and accidental venous intervention rate.

Results: Average intervention time (26 (21-25) vs 39 (25-61) min; $\mathrm{p}<0.001)$, average number of intervention attempts $(1.10 \pm 0.35 \mathrm{vs} .1 .58 \pm 1.05 ; \mathrm{p}=0.012)$, average accidental venous intervention rate $[3(1.9 \%)$ vs. $16(10 \%) ; \mathrm{p}<0.001]$, and average visual analog scale score for pain $(2(1-5)$ vs. 7 (3-9); $\mathrm{p}<0.001)$ were significantly lower in group 1. Success rate at initial intervention was higher in group 1 [140 (89\%) vs. 105 (69\%); p<0.001]. Frequency of hematoma, early hematoma, and arteriovenous fistula were lower in group 1.

Conclusions: Ultrasound-guided femoral artery intervention requires less time and provides higher cannulation success rate at the initial attempt, with lesser pain and lower complication rates.
\end{abstract}

Keywords: Complications; Geriatrics; Intervention; Palpation; Ultrasound.

Cite This Article: Hasan Ata Bolayir, Hakan Gunes, and Muratkerkutluoglu. (2018). "WHAT IS THE MOST APPROPRIATE METHOD FOR COMMON FEMORAL ARTERY CANNULATION IN PATIENTS AGED ABOVE 75 YEARS WHICH IS THE MOST APPROPRIATE METHOD." International Journal of Research - Granthaalayah, 6(11), 431-438. https://doi.org/10.29121/granthaalayah.v6.i11.2018.1149. 


\section{Introduction}

The physiopathology of atherosclerosis coincides with the physiology of aging at several points. As a result, both peripheral and coronary artery diseases frequently occur in geriatric patients. The diagnosis and treatment of coronary and peripheral artery diseases in geriatric patients decrease both mortality and morbidity and improve quality of life. The gold standard in the diagnosis and treatment of coronary and peripheral artery diseases is angiography. Although the preference of transradial intervention has increased to reduce complications in vascular intervention sites and provide patient comfort, in all patient groups, the femoral artery is frequently used as the vascular intervention site in the diagnosis and treatment of coronary and peripheral artery diseases (1).Local vascular complications are the most frequent complications in angiographic procedures and are affected by various factors such as operation experience, gender, overweight or underweight, renal failure, and anticoagulant or antiaggregant used (2-5). The most commonly observed complications are local hematoma, retroperitoneal hemorrhage, pseudoaneurysm (PA), arteriovenous fistula, infection, and other local injuries (5). In geriatric patients that have especially poor vascular structure, these complications occur frequently, and it is more difficult to deal with them compared with the other age groups. The reduction inthese complications in the geriatric population will result in a significant decrease in mortality and morbidity.

The site of femoral artery puncture is the medial area between the inguinal ligament and the lower end of the femoral head. If the intervention is performed below the inguinal ligament, the development of aneurysms and arteriovenous fistulas increase, whereas if it is performed above the inguinal ligament, the risk of retroperitoneal bleeding increases $(6,7)$ In order to reduce such complications, the use of ultrasound (US)-guided artery and venous interventions have increased. US-guided intervention is frequently used in venous interventions. Multiple randomized studies and two meta-analyses showed that US-guided venous interventions reduced complications, intervention time, and number of intervention attempts $(8,9)$. On the other hand, there are a limited number of studies evaluating the complications of US-guided arterial interventions, and the effect of US on complications in intervention sites, pain scores, and number of intervention attempts has never been evaluated, especially in the geriatric population.

In this study, we aimed to compare the intervention site complications, number of attempts for artery cannulation, intervention times, success rate at initial intervention, accidental femoral vein intervention rates, and pain score in US-guided interventions and conventional methods in femoral artery interventions performed on geriatric patients in whom angiography is required.

\section{Materials and Methods}

This was a prospective randomized controlled study. Patients aged $>75$ years who were taken to the catheterization laboratory for diagnostic or therapeutic intervention via femoral artery were consecutively included in the study, and those who underwent manual intervention (manual group) and US-guided intervention (US group) to the common femoral artery were grouped accordingly. Informed consent was obtained from all patients.

The researchers were separated into two groups: those who were experienced in manual intervention (performing more than 1000 operations per year) and those who were experienced in 
US-guided intervention (performing more than 1000 operations per year); both groups of researchers only performed the intervention techniques in which they are experienced.

The Venue 40 Ultrasound system (Antares, Siemens Erlangen Germany) was used; a 9-13 MHz linear transducer was utilized.

In all patients, the modified Seldinger technique was used as the intervention technique. Researchers using the manual method, after the application of local anesthetics, marked the center of the femoral head with a clamp under fluoroscopy and used it as a marker to cannulize the common femoral artery (CFA). Similarly, for US-guided CFA cannulization, local anesthetics were applied around the artery. During the US-guided CFA cannulization, the US probe was placed on the left side and in the left hand of the operator, while an 18-gauge needle was placed in the right hand. In this way, the operator was able to simultaneously manipulate both the US probe and the needle.

Number of artery puncture attempts by the operator to place the sheath and accidental vein punctures were recorded by an independent observer. Each attempt was defined as removing the needle and making a new puncture. Intervention time was determined by the laboratory staff as the time spent from the initial movement of the table for fluoroscopy or US application until the successful placement of the sheath. Recorded time did not include the time needed for preparing the US probe. Vascular sheath size was between 5 Fr and 7 Fr depending on the requirements for angiography or interventional operation. Antiaggregant and anticoagulant treatments received by all patients during the operation or within $24 \mathrm{~h}$ postoperatively were recorded.

The intensity of pain in all patients was recorded during the operation using the visual analog scale (VAS). The severity of pain according to VAS was graded as 0 for "no pain" and 10 for "worst pain imaginable" (10-cmscale). The score was noted by the laboratory nurse by investigating the patient. During sheath removal postoperatively, manual compression and pressure exertion were used.

During the operation, the sheath was removed when the active coagulation time decreased to $<180$ $\mathrm{s}$ in patients who were administered fractionated heparin during the coronary or peripheral treatment operation. The sheath was removed $15 \mathrm{~min}$ postoperatively in patients who only underwent diagnostic operation. All patients were followed up for complications that may arise before hospital discharge. Patients who were discharged within 7 days postoperatively were followed up again for possible complications after discharge.

Pseudoaneurysm formation, retroperitoneal hemorrhage, arteriovenous fistula, hematoma formation $>5 \mathrm{~cm}$, and early hematoma formation during sheath placement were recorded as complications. These complications were recorded during discharge and 7 days postoperatively by the operator who performed the operations on the patients through follow-upsat least two more times.

The study was conducted in accordance with the Helsinki Declaration of Human Rights and was approved by the ethics board of our hospital. 


\section{Statistical Analysis}

Statistical analyses were conducted via SPSS 17.0 (for Windows SPSS 17.0, Chicago, Illinois). Continuous variables were presented as average \pm standard deviation (for parameters with normal distribution) and median (25/75\% interquartile range) for parameters without normal distribution), and categorical variables were presented as percentages. Comparison of categorical variables between the groups was performed using the chi-square test. Normality analysis was performed using the Kolmogorov-Smirnov test. The Student's t-test was used for the comparison of normally distributed variables, and the Mann-Whitney U test was used for the comparison of normally distributed multiple variables. A two-tailed $\mathrm{p}$ value of $<0.05$ was determined to be statistically significant within a confidence interval of $95 \%$.

\section{Results}

309 patients were included in the study with 157 in the US group and 152 in the manual group. The average age in the US group was 79(76-84) years, and the female to male ratio was 51/106. The average age in the manual group was 79(76-85) years, and the female to male ratio was 47/105; both groups were similar in terms of age and gender distribution $(p=0.626$ and $p=0.863$, respectively). The main characteristics parameters of the patients are shown in Table 1.

Table 1: Laboratory, Demographic, and Clinical Characteristics of the Groups

\begin{tabular}{|l|l|l|l|}
\hline & US group (n=157) & Manual group (n=152) & P value \\
\hline Age (years) & $77.5 \pm 3.5$ & $83.2 \pm 4.1$ & 0.096 \\
\hline Weight (kg) & $71.7 \pm 10.8$ & $70.1 \pm 10.2$ & 0.168 \\
\hline Height (cm) & $159.7 \pm 5.5$ & $165 \pm 7.1$ & 0.051 \\
\hline Female/male (n) & $51 / 106$ & $47 / 105$ & 0.863 \\
\hline Hypertension (n, \%) & $40(25 \%)$ & $33(21 \%)$ & 0.768 \\
\hline Hyperlipidemia (n, \%) & $38(24 \%)$ & $47(30 \%)$ & 0.187 \\
\hline Diabetes mellitus (n, \%) & $30(19 \%)$ & $36(23 \%)$ & 0.232 \\
\hline Smoking (n, \%) & $60(38 \%)$ & $57(37 \%)$ & 0.897 \\
\hline 7-Fr Sheath size (n, \%) & $9(6 \%)$ & $4(3 \%)$ & 0.169 \\
\hline Acetylsalicylic acid (n, \%) & $43(27 \%)$ & $33(21 \%)$ & 0.249 \\
\hline Clopidogrel (n, \%) & $29(18 \%)$ & $37(24 \%)$ & 0.209 \\
\hline UFH (n, \%) & $24(15 \%)$ & $36(23 \%)$ & 0.063 \\
\hline Enoxaparin (n, \%) & $2(1 \%)$ & $3(2 \%)$ & 0.299 \\
\hline Tirofiban (n, \%) & $1(0.6 \%)$ & $1(0.6)$ & 0.863 \\
\hline Abciximab (n, \%) & 0 & $1(0.6)$ & 0.896 \\
\hline Interventional procedure (n, \%) & $26(16 \%)$ & $39(25 \%)$ & 0.061 \\
\hline Two-sided intervention & $1(0.6 \%)$ & $6(3.9 \%)$ & 0.051 \\
\hline Laboratory findings & & & \\
\hline BUN (mg/dl) & $23 \pm 14$ & $21 \pm 11$ & 0.112 \\
\hline Creatinine (mg/dL) & $1.15 \pm 0.5$ & $1.14 \pm 0.74$ & 0.911 \\
\hline Sodium & $137 \pm 3.5$ & $137 \pm 3.6$ & 0.931 \\
\hline Potassium & $4.3 \pm 0.6$ & $4.1 \pm 0.5$ & 0.729 \\
\hline Triglyceride (mg/dL) & $145 \pm 116$ & $145 \pm 129$ & 0.983 \\
\hline HDL (mg/dL) & $34 \pm 9$ & $36 \pm 17$ & \\
\hline
\end{tabular}




\begin{tabular}{|l|l|l|l|}
\hline TOTAL $(\mathrm{mg} / \mathrm{dL})$ & $172 \pm 45$ & $166 \pm 49$ & 0.287 \\
\hline LDL $(\mathrm{mg} / \mathrm{dL})$ & $111 \pm 33$ & $109 \pm 32$ & 0.553 \\
\hline Hemoglobin $(\mathrm{g} / \mathrm{dL})$ & $13.9 \pm 2.0$ & $14.4 \pm 2.2$ & 0.986 \\
\hline Platelet $\left(\times 10^{3} / \mu \mathrm{L}\right)$ & $267 \pm 71$ & $228 \pm 70$ & 0.233 \\
\hline Leukocytes $(\mathrm{n} / \mu \mathrm{L})$ & $12.4 \pm 3.6$ & $9.1 \pm 3.2$ & 0.134 \\
\hline
\end{tabular}

Abbreviations: BUN:blood urea nitrogen, HDL: high density lipoprotein, TOTAL: total cholestrol, LDL: low density lipoprotein.

There were no statistically significant differences between the groups in terms of age, gender, hypertension, diabetes, smoking, and hyperlipidemia or antiagregan usage before the procedure. The 7Fr sheath was frequently used in the US group. However, this ratio was not statistically significant. When the characteristics of the procedures were compared, average intervention time (26(21-25) vs 39(25-61)min; $\mathrm{p}<0.001)$, number of intervention attempts $(1.10 \pm 0.35$ vs. 1.58 $\pm 1.05 ; \mathrm{p}=0.012$ ), vein puncture ratio [3(1.9\%) vs. $16(10 \%) ; \mathrm{p}<0.001]$, and average VAS pain score $(2(1-5)$ vs. $7(3-9) ; p<0.001)$ were found to be significantly lower in the US group compared with the manual group. In addition, the success rate at the initial attempt was significantly higher in the US group than in the manual group [success rate n: $140(89 \%)$ vs. $105(69 \%)$; $\mathrm{p}<0.001$; Table 2].

Table 2: Comparison of procedural characteristics between the groups

\begin{tabular}{|l|l|l|l|}
\hline & $\begin{array}{l}\text { USgroup } \\
(\mathbf{n = 1 5 7})\end{array}$ & $\begin{array}{l}\text { Manual group } \\
(\mathbf{n = 1 5 2})\end{array}$ & P value \\
\hline Average intervention time (s) & $34.5 \pm 41.3$ & $42.5 \pm 68.7$ & 0.013 \\
\hline $\begin{array}{l}\text { Success rate at initial intervention }(\mathbf{n}, \\
\text { \%) }\end{array}$ & $140(89 \%)$ & $105(69 \%)$ & $<0.001$ \\
\hline Vein puncture (n, \%) & $3(1.9 \%)$ & $16(10 \%)$ & $<0.001$ \\
\hline $\begin{array}{l}\text { Number of attempts for intervention } \\
\text { (n) }\end{array}$ & $1.10 \pm 0.35$ & $1.58 \pm 1.05$ & 0.012 \\
\hline VAS Pain score & $2.9 \pm 2.2$ & $5.9 \pm 2.9$ & $<0.001$ \\
\hline
\end{tabular}

Abbreviations: VAS: visual analog scale.

When operation complications were evaluated, early hematoma and AV fistula were found to be significantly less frequent in the US group. There were no significant differences between the two groups in terms of the frequencies of PA and major bleeding (Table 3).

Table 3: Comparison of Complications Rates Between The Groups

\begin{tabular}{|l|l|l|l|}
\hline Complications & USgroup $(\mathbf{n}=157)$ & Manualgroup $(\mathbf{n = 1 5 2})$ & P value \\
\hline Early hematoma (n, \%) & $0(0)$ & $6(3.9 \%)$ & 0.012 \\
\hline Hematoma (n, \%) & $4(2.5 \%)$ & $14(9.2 \%)$ & 0.013 \\
\hline AV Fistula (n, \%) & $1(0.6 \%)$ & $6(3.9 \%)$ & 0.040 \\
\hline Pseudo aneurysm (n, \%) & $1(0.6 \%)$ & $1(0.7 \%)$ & 0.165 \\
\hline Major bleeding (n, \%) & $0(0)$ & $1(0.7 \%)$ & 0.348 \\
\hline
\end{tabular}




\section{Discussion}

In this study, we demonstrated that US-guided femoral artery puncture in patients aged $>75$ years resulted in less vein punctures, less time, less attempts, and higher success rate at the initial intervention compared with the conventional method.

The most frequent complication during femoral artery puncture is hematoma (10). Early hematoma occurs after sheath placement and is usually an indicator of artery laceration. Late hematoma occurs within $12 \mathrm{~h}$ after sheath removal (11).In patients who were treated with US-guided femoral artery puncture, the occurrence of both early and late hematomas is less frequent because of lesser attempts and higher success rates at the initial attempt. Arteriovenous (AV) fistulas occur if there is de-puncture to the femoral vein during the puncturing of the femoral artery or if continuous bleeding occurs after the artery puncture is drained into the vein $(11,12)$. Reduction or complete avoidance of femoral vein punctures will decrease the probability of AV fistula. In our study, the occurrence of AV fistulas in the group treated with US-guided femoral artery puncture was lower compared with the occurrence in the group treated via artery puncture with the conventional method. One of the main risk factors for the development of PA is superficial femoral artery (SFA) intervention $(13,14)$. In our study, because there were fewer or no SFA interventions in the group treated via US-guided femoral artery puncture, the rate of PA occurrence was lower. Manual compression is the first treatment step in frequent major vascular complications that occur in angiography operations such as hematoma and PA. Both mechanical pains and secondary pains of osteoporosis are frequent in geriatric patients; long-term immobility and mechanical compression increase the severity of pain and make it more difficult to handle complications. Therefore, it is crucial to prevent complications especially in geriatric patients. In this study,severity of pain in patients during the puncture was evaluated using the VAS pain scale;it was found that US-guided femoral artery puncture resulted in less pain.

The largest study conducted with a design similar to ours was the FAUST study by Seto et al. (15) this study showed that when US-guided artery cannulation is compared to the manual method, USguided cannulation was more successful and the rate of complications was lower. However, because the average age of the patient groups was $64.2 \pm 11$.4years and63.5 \pm 12.4 years in the conventional and US groups, respectively, the study is not applicable to the geriatric patient group.

In a smaller scale study conducted by Gedikoglu et al., in line with the findings of our study, the success rate at initial intervention, sheath placement time, and intervention time were found to be superior in the US group compared with the manual group. Only superficial hematoma was evaluated with respect to local complications; however, no significant difference was found between the two groups ( $\mathrm{p}=0.052$ ).In our study, local complications were found to be lower in the US group, and the difference was statistically significant. Again, this study is not fully applicable to the geriatric patient group (16).

Although the use of US for intervention is a time-consuming process for angiography units with high patient population, preparation of the device in standby takes $1 \mathrm{~min}$ on an average (16). While certain training is required for the use of US, it takes less time for cardiologists trained in echocardiography to learn. Once US use becomes frequent in artery punctures, the decrease in complications of intervention site in US may render US to be a routine part of angiography, 
especially in the geriatric population. It is known that US use is successful in difficult cases such as percutaneous treatment of abdominal aorta or in cases where in popliteal and infrapopliteal arteries are totally blocked and there is no anterograde transport (17-19).

Because our study was conducted by experienced physicians who conduct $>1000$ cases per year, these data and complication ratios are definitely not applicable to physicians with less experience. Again, because the patients included in our study were randomized without femoral artery palpation by the operator, complications might have been lesser and success ratio might have been higher in the US group. Use of a second observer and laboratory timer may have increased the pressure on the operators performing the puncture using the conventional method and decreased their probability of success.

\section{Conclusion}

In conclusion, according to the results of this study, US-guided CFA cannulation provides higher success rates for cannulation in lesser time and at the initial attempt while causing less pain and complications. This is very important for the prevention of complications and patient comfort in geriatric patients who are more sensitive and fragile because of their age.

Acknowledgements: No acknowledgements.

No Conflict of Interest: The authors declare any conflict of interest.

\section{References}

[1] Feldman, D. N., Swaminathan, R. V., Kaltenbach, L. A., Et Al (2013). "Adoption of Radial Access and Comparison of Outcomes To Femoral Access In Percutaneous Coronary Intervention Clinical Perspective: An Updated Report From The National Cardiovascular Data Registry (2007-2012) ". Circulation, 127(23), 2295-2306.

[2] Corley, J. A., Kasliwal, M. K., Tan, L. A., Et Al. (2014). "Delayed Vascular Claudication Following Diagnostic Cerebral Angiography: A Rare Complication of The Angioseal Arteriotomy Closure Device". Journal of Cerebrovascular and Endovascular Neurosurgery, 16(3), 275-280.

[3] Patel, M. R., Jneid, H., Derdeyn, C. P., Et Al. (2010). "Arteriotomy Closure Devices for Cardiovascular Procedures: A Scientific Statement from The American Heart Association". Circulation, 122(18), 1882-1893.

[4] Chandrasekar, B., Doucet, S., Bilodeau, L.,Et Al. (2001). "Complications Of Cardiac Catheterization In The Current Era: A Single-Center Experience". Catheterization And Cardiovascular Interventions, 52(3), 289-295.

[5] Sherev, D. A., Shaw, R. E., \& Brent, B. N. (2005). "Angiographic Predictors Of Femoral Access Site Complications: Implication For Planned Percutaneous Coronary Intervention". Catheterization And Cardiovascular Interventions, 65(2), 196-202.

[6] Altin, R. S., Flicker, S., Naidech, H. J. (1989). "Pseudoaneurysm And Arteriovenous Fistula After Femoral Artery Catheterization: Association With Low Femoral Punctures". American Journal Of Roentgenology, 152(3), 629-631.

[7] Kim, D., Orron, D. E., Skillman, J. J., Et Al. (1992). "Role of Superficial Femoral Artery Puncture In The Development Of Pseudoaneurysm And Arteriovenous Fistula Complicating Percutaneous Transfemoral Cardiac Catheterization". Catheterization and Cardiovascular Diagnosis, 25(2), 9197. 
[8] Hind, D., Calvert, N., Mcwilliams, R., Et Al. (2003). "Ultrasonic Locating Devices For Central Venous Cannulation: Meta-Analysis". Bmj, 327(7411), 361.

[9] Randolph, A. G., Cook, D. J., Gonzales, C. A., Et Al. (1996). "Ultrasound Guidance for Placement Of Central Venous Catheters: A Meta-Analysis Of The Literature". Critical Care Medicine, 24(12), 2053-2058.

[10] Feldman, T. (1998). "Percutaneous Vascular Closure: Plugs, Stitches, And Glue". Catheterization and Cardiovascular Diagnosis, 45(1), 89-89.

[11] Samal, A. K., White, C. J. (2002). "Percutaneous Management Of Access Site Complications". Catheterization And Cardiovascular Interventions, 57(1), 12-23.

[12] Sidawy, A. N., Neville, R. F., Adib, H., Et Al (1993). "Femoral Arteriovenous Fistula Following Cardiac Catheterization: An Anatomic Explanation". Cardiovascular Surgery, 1(2), 134-137.

[13] Webber, G. W., Jang, J., Gustavson, S., Et Al. (2007). "Contemporary Management of Postcatheterization Pseudoaneurysms". Circulation, 115(20), 2666-2674.

[14] Katzenschlager, R., Ugurluoglu, A., Ahmadi, A., Et Al. (1995). "Incidence of Pseudoaneurysm after Diagnostic and Therapeutic Angiography". Radiology, 195(2), 463-466.

[15] Seto, A. H., Abu-Fadel, M. S., Sparling, J. M., Et Al. (2010). "Real-Time Ultrasound Guidance Facilitates Femoral Arterial Access and Reduces Vascular Complications: Faust (Femoral Arterial Access with Ultrasound Trial) ". Jacc: Cardiovascular Interventions, 3(7), 751-758.

[16] Gedikoglu, M., Oguzkurt, L., Gur, S.,Et Al. (2013). "Comparison of Ultrasound Guidance with The Traditional Palpation and Fluoroscopy Method for The Common Femoral Artery Puncture". Catheterization and Cardiovascular Interventions, 82(7), 1187-1192.

[17] Oguzkurt, L., Gürel, K., Eker, E., Et Al. (2012). "Ultrasound-Guided Puncture of the Femoral Artery for Total Percutaneous Aortic Aneurysm Repair". Diagnostic and Interventional Radiology, 18(1), 92.

[18] Arthurs, Z. M., Starnes, B. W., Sohn, V. Y., Et Al. (2008). "Ultrasound-Guided Access Improves Rate Of Access-Related Complications For Totally Percutaneous Aortic Aneurysm Repair". Annals of Vascular Surgery, 22(6), 736-741.

[19] Gür, S., Oguzkurt, L., Gürel, K.,Et Al. (2013). "Us-Guided Retrograde Tibial Artery Puncture For Recanalization Of Complex Infrainguinal Arterial Occlusions". Diagnostic and Interventional Radiology, 19(2), 134.

*Corresponding author.

E-mail address: habolayir@ hotmail.com 